\title{
Evolution of the microstructure during the process of consolidation and bonding in soft granular solids
}

\author{
B. Yohannes ${ }^{\mathrm{a}}$, M. Gonzalez ${ }^{\mathrm{b}}$, A. Abebec ${ }^{\mathrm{c}}$, O. Sprockel ${ }^{\mathrm{c}}$, F. Nikfar , S. \\ Kiang $^{\mathrm{d}}$, A. M. Cuitiño ${ }^{\mathrm{a}, *}$ \\ ${ }^{a}$ Mechanical and Aerospace Engineering, Rutgers University, Piscataway, NJ 08854 \\ ${ }^{b}$ School of Mechanical Engineering, Purdue University, West Lafayette, IN 47907 \\ ${ }^{c}$ Bristol-Myers Squibb Company, Drug Product Science and Technology, New Brunswick, \\ NJ 08903 \\ ${ }^{d}$ Chemical and Biochemical Engineering, Rutgers University, Piscataway, NJ 08854 \\ (formerly at Bristol-Myers Squibb Company, New Brunswick, NJ 08903)
}

\begin{abstract}
The evolution of microstructure during powder compaction process was investigated using a discrete particle modeling, which accounts for particle size distribution and material properties, such as plasticity, elasticity, and interparticle bonding. The material properties were calibrated based on powder compaction experiments and validated based on tensile strength test experiments for lactose monohydrate and microcrystalline cellulose, which are commonly used excipient in pharmaceutical industry. The probability distribution function and the orientation of contact forces were used to study the evolution of the microstructure during the application of compaction pressure, unloading, and ejection of the compact from the die. The probability distribution function reveals that the compression contact forces increase as the compaction force increases (or the relative density increases), while the
\end{abstract}

\footnotetext{
${ }^{*}$ Corresponding author

Email address: cuitino@jove.rutgers.edu, Tel.:+1 8484454210 (A. M. Cuitiño ) 
maximum value of the tensile contact forces remains the same. During unloading of the compaction pressure, the distribution approaches a normal distribution with a mean value of zero. As the contact forces evolve, the anisotropy of the powder bed also changes. Particularly, during loading, the compression contact forces are aligned along the direction of the compaction pressure, whereas the tensile contact forces are oriented perpendicular to direction of the compaction pressure. After ejection, the contact force becomes isotropic.

Keywords: powder compaction; discrete particle modeling; microstructure anisotropy; tablets; tensile strength; force distribution;

\section{1. Introduction}

2 Powder compaction is widely used in manufacturing, such as pharma3 ceutical industries, to produce tablets of high relative density and strength. ${ }_{4}$ To get high quality design and product, understanding the mechanics of the 5 compaction process is essential. During compaction, the particles deform 6 significantly and usually develop bonding at contact surfaces. The amount 7 of deformation of each particle and the bond strength created between con8 tacting particles depends on the properties of the particles and the applied 9 compaction pressure.

10 A lot has been learned from prior experimental studies on tablet com11 paction, including the effects of particle size on powder compaction (Adolf12 sson et al., 1997; Almaya and Aburub, 2008; Eichie and Kudehinbu, 2009; 13 Fichtner et al., 2005; Herting and Kleinebudde, 2007; Khomane and Bansal, 14 2013; Mckenna and Mccafferty, 1982; Patel et al., 2007; Sun and Himmelspach, 
2006; Sun, 2008; Yohannes et al., 2015), the role of lubricants on compaction and tensile strength of tablets (Almaya and Aburub, 2008; Sun and Himmelspach, 2006), relationship between porosity and compaction pressure (Heckel, 1961) to mention a few. However, experiments alone cannot provide full description of the characteristics in the bulk of the compacted powders. To understand and investigate the compaction process in detail, particularly the interactions between individual particles and the heterogeneity in the bulk, computational modeling of the compaction process is required. Various methods have been used to model powder compaction in the past. These methods are based on Finite Element Method (FEM) (Klinzing et al., 2010), Discrete Element Method (DEM) (Cundall and Strack, 1979; Koynov et al., 2011; Martin and Bouvard, 2004; Olsson and Larsson, 2013; Sheng et al., 2004; Skrinjar and Larsson, 2004), or combined Discrete-Finite Element Method (DFEM) (Zavaliangos, 2002; Zhang, 2009; Zhang and Zavaliangos, 2011; Zheng and Cuitiño, 2002). In FEM, usually, only the global values of the physical properties are assumed, while in DEM the interaction between individual particles are accounted for, which enables modeling the heterogeneity of powders at the microscopic/particle scale. Hence, DEM gives more accurate results than continuum methods, which consider only the global physical properties of the material. In DEM, the inter-particle forces are computed based on the physical properties of the particles. Originally, DEM was developed for application pertaining to very small elastic deformations (Cundall and Strack, 1979). In recent years, DEM has been used to model plastic deformation (Martin and Bouvard, 2004; Olsson and Larsson, 2013; Sheng et al., 2004) and bonding between particles (Potyondy 
and Cundall, 2004).

In DEM, an explicit integration method is usually used to track the displacement and deformation of individual particles at every time step. Since DEM accounts for the interaction of individual particles directly, it requires a significantly large computational resources, especially for large number of particles. To reduce the computational time requirement, Sheng et al. (2004) increased the time step in the numerical integration by increasing the mass of individual particles by a factor of 10,000. Using this approach, the same authors showed that for a given compaction pressure, the strain decreases as the coefficient of friction between the particles is increased. In addition, they showed that lower coefficient of friction of particle results in higher coordination number (number of contact between particles). Similarly, Olsson and Larsson (2013), using DEM simulations of binary mixtures, showed that the particle size distribution has a very small influence on the compaction pressure and elastic unloading for narrow particle size distribution (Martin and Bouvard, 2004).

At larger deformations, the shape of particles deviates significantly from a spherical shape and the inter-particle contact forces depend on each other, in addition to the material property, effectively exhibiting a non-local effect (Gonzalez and Cuitiño, 2012). Further, Mesarovic and Johnson (2000) used FEM models to study details of deformation mechanisms of particles. To account for these complicated particle deformation and contact forces, Zavaliangos (2002) has used DFEM, which can be considered as an advanced DEM, because in addition to computing the contact force between two particles, the deformation of the particles is further modeled using a finite element 
method. Specially, for compaction of powder bed to a very large relative densities, the particles deform significantly and only considering the assumptions of the DEM will not be sufficient. However, DFEM requires huge computational resource compared to DEM and is mostly applied to 2D simulations with limited number of particles, though it can be applied to 3D simulations as well (Güner et al., 2015).

In this paper, a 3D numerical compaction of granular materials was used (Gonzalez and Cuitiño, 2012, 2015; Martin and Bouvard, 2004; Mesarovic and Johnson, 2000; Storåkers et al., 1997, 1999). This method has a reduced computational time compared to DEM, while accounting for the interaction of particles directly as in the case of DEM. Based on this method and a compaction experiment, the material properties of two commonly used pharmaceutical powders were calibrated. The simulations were validated by compacting the powders at various compaction pressures and measuring the diametrical tensile strength. Then, the evolution of the microstructure during compaction, unloading, and ejection of the compacts were analyzed. Specifically, the evolution of the probability distribution of the contact forces, the anisotropy, and the inter-particle bond strength during the compaction process were investigated.

\section{Numerical Simulation}

The first step in all kinds of discrete particle modeling schemes is forming a packing of particles with the target particle size distribution. For this study, the particle size distribution of two pharmaceutical powders, lactose monohydrate (Foremost Farms, Baraboo, WI) and microcrystalline cellulose (MCC- 
Avicel PH102, FMC Biopolymer, Newark, DE) were used. Since discrete particle modeling methods require the representation of individual particles in the material, the computational efficiency is constrained by the number of particles in the system. State-of-the-art discrete particle models are capable of modeling about a million particles. However, even a very small volume of powder bed consists of a vast number of particles. For example, a $500 \mathrm{mg}$ of MCC-Avicel PH-102 (MCC) powder consists of about 9 billion particles. In terms of number of particles, the majority of these particles (about 91\%, calculated based on measured particle size distribution (Yohannes et al., 2015) and assuming all particles are spherical) are fine particles of less than $1 \mu \mathrm{m}$ size. In terms of volume, these fine particles account for about only $3 \%$ of the total volume. On the other hand, if the particle sizes less than $100 \mu \mathrm{m}$ are removed, a $500 \mathrm{mg}$ of MCC powder will have only about 500,000 particles. Our previous experiments (Yohannes et al., 2015) have indicated that removing the fine particles does not affect the compaction profile and tensile strength of the compacts. Fig. 1 shows the compaction profiles, during unloading and loading of the compaction pressure, for four samples of lactose powder with differing amount of fine particles. The four samples were compacted to in-die maximum relative densities of 0.90 and 0.97 , which correspond to maximum compaction pressures of $150 \mathrm{MPa}$ and $230 \mathrm{MPa}$, respectively. The four samples have very similar compaction profiles indicating that the fine particles have little or no effect on the compaction of the samples. Based on these results, it is possible to use particle sizes greater than $100 \mu \mathrm{m}$ to model the powder compaction, which will significantly reduce the number of particles used in the simulations.(These compaction profiles are later used 
to calibrate the mechanical properties of the powders). Fig. 2 shows the particle size distribution used in the simulations and experiments in terms of number of particles $\left(f_{o}(\mathrm{~d})\right)$. The procedures for the preparation of the sieved powder samples are discussed in detail in Yohannes et al. (2015). The sizes of particles range between 100 and $240 \mu \mathrm{m}$ for lactose and between 100 and $750 \mu \mathrm{m}$ for MCC.

These particles were deposited in a cylindrical die using a ballistic gravitational deposition method (Bratberg et al., 2002; Jullient et al., 1992; Koynov et al., 2011; Mueller, 1997). In this ballistic deposition method only the static equilibrium of individual particles is used as a criteria, while the others factors that may affect packing of granular materials, such as the dynamic forces, friction, and cohesion are ignored. A particle is allowed to roll down on other particles freely until it locks into a stable configuration. To be in stable configuration (or static equilibrium) in 3-D systems, a frictionless particle should be in contact with at least three other particles/walls such that motion or rolling in any direction is prohibited due to the contacts with the neighboring particles. A particle can also be in equilibrium if it lies on a horizontal plane wall. This ballistic deposition method is much faster that other gravitation deposition methods that consider the dynamic contact forces and integration time steps. Fig. 3.a shows deposited lactose particles. The particles were deposited in a 3mm-diameter die and the total number particle is about 3000 and 2500 for lactose and MCC, respectively.

In the experiments, the initial relative densities for lactose and MCC powders were 0.47 and 0.34 , respectively (Yohannes et al., 2015). These values are very small compared to theoretical possible relative densities for 
pack of spherical particles. The lower initial density in the experiment can be attributed to the irregular shape of the particles (Yohannes et al., 2015; Zou and $\mathrm{Yu}, 1996)$ and inter-particle attractive force developed in the presence of small particles (Castellanos, 2005). In the simulations, only spherical particles were used and the inter-particle attractive force was ignored. The initial relative density in the simulations for both lactose and MCC was about 0.51. The initial relative density using this deposition method is closer to that of the experiments, compared to other particle deposition methods.

After the particles are deposited, a compaction pressure is gradually applied through upper and lower punches. The compaction simulation is a strain controlled simulation, where a small displacement is applied to the two punches. The pressure on the punches is then measured based on the contact forces from the particles. The contact forces are discussed in detail in the next section. After the powder bed was compacted to the target relative density (also referred to as solids fraction), the punches are gradually removed. Finally, the compacted powder is ejected from the die by gradually pushing the compact using the lower punch. Fig. 3.b shows lactose powder after compaction.

During the compaction, unloading, and ejection simulation, the contact force between the particles is computed based on the deformation and mechanical properties of the particles. The deformation is computed as the overlap between pair of contacting particles. The plastic properties, elastic properties, and bonding parameters of the particles are also used to compute the force. A pair of contacting particles can be in a loading mode (where the particles are in contact for the first time), or unloading mode (where the 
contact force on a pair of contacting particles is decreasing), or reloading mode (where a previously contacting particles are overlapping again). During loading mode, the particles are restricted to follow a power-law plasticity model (Martin and Bouvard, 2004; Mesarovic and Johnson, 2000; Storåkers et al., 1997, 1999),

$$
\sigma=k \epsilon^{1 / m},
$$

where $k$ and $m$ are particle plastic parameters, $\epsilon$ and $\sigma$ are strain and stress of the particles, respectively. The contact force $(P)$ between two particles is computed as

$$
P=k_{p} a^{2+1 / m},
$$

where $a$ is the radius of the contact area between the pair of contacting particles $a=\sqrt{2 c_{2} \frac{\gamma}{\frac{1}{R_{1}}+\frac{1}{R_{2}}}}, \gamma$ is the overlap between two contacting particles, and $m$ is the average plastic parameter, $m=\left(m_{1}+m_{2}\right) / 2$ (the subscripts refer to the pair of contacting particles). The parameter $k_{p}$ is given as

$$
k_{p}=K \pi\left(\frac{1}{k_{1}^{m}}+\frac{1}{k_{2}^{m}}\right)^{-\frac{1}{m}}\left(\frac{1}{R_{1}}+\frac{1}{R_{2}}\right)^{\frac{1}{2 m}-1}\left(2 c_{2}\right)^{1+\frac{1}{2 m}},
$$

where $R$ is the radius of the particles, $K=3 \times 6^{-\frac{1}{m}}, c_{2}=1.43 \exp \left(-\frac{0.97}{m}\right)$.

During the unloading of the contact force, $P$ is computed as,

$$
P=\frac{2 k_{p}}{\pi} a_{p}^{2+1 / m}\left[\arcsin \left(\frac{a}{a_{p}}\right)-\frac{a}{a_{p}} \sqrt{1-\left(\frac{a}{a_{p}}\right)^{2}}\right]-\sqrt{8 \pi \omega E_{m}} a_{p}^{3 / 2}\left(\frac{a}{a_{p}}\right)^{3 / 2},
$$

where $a_{p}$ is the radius of the contact area at the maximum $P$ during the loading phase, $\omega$ is the bonding energy per area, and $E_{m}$ is the effective 
Young's modulus,

$$
E_{m}=\left(\frac{1-\nu_{1}^{2}}{E_{1}}+\frac{1-\nu_{2}^{2}}{E_{2}}\right)^{-1},
$$

where $E$ is the Young's modulus, $\nu$ is the Poisson Ratio.

The material properties of the particles in the simulation were calibrated by comparing the compressibility of the powders (compaction pressure vs. relative density curve) of the simulations to that of experiments. In the experiments, a Presster machine (The Metropolitan Computing Corporation of East Hanover, NJ) equipped with a $10 \mathrm{~mm}$ flat face round tooling was used to compact the powders (Yohannes et al., 2015). During the application of the compaction pressure, the plastic parameters play a significant role compared to the elastic and bonding parameters. Therefore, the plastic parameters $k$ and $m$ are calibrated using the compaction pressure vs. relative density curve during the application of the compaction pressure. Fig. 4 shows the comparison of several simulation results with an experimental result. Initially (up to relative density of 0.55 ), only the position of the particles is rearranged without pressure increase on the punches and die wall. Beyond relative density of 0.55 , the pressure on the punches increases steadily. To select the appropriate values of the plastic parameters, several combination of the plastic parameters $k$ and $m$ were tested. The tested values range from $0.15-3.75 \mathrm{GPa}$ for $k$ and from 1.0 - 4.0 for $m$. As shown in the figure, increasing $k$ and $m$ increases the compaction pressure for a given relative density. The best fitting combination of $k$ and $m$ was chosen by comparing the least mean square values. Table 1 shows the material properties selected for lactose and MCC powders based on the lowest least mean square value. The difference between the simulation and experimental results are more no- 
ticeable at the early stage of compaction. This difference is associated with the difference of the initial relative densities in the simulations and experiments during deposition of the powder in the die, as mentioned earlier. As the compaction pressure is increased the difference between simulation and experimental results decrease, with an error of 1-10\% above relative density of 0.80 .

Once the parameters $k$ and $m$ were selected, the effective Young's modulus $\left(E_{m}\right)$ and the bonding energy $(\omega)$ can be calibrated using the compaction vs. relative density curve during the unloading of the compaction pressure. Fig. 5 shows comparison of several simulation results with experimental results for lactose. Several combinations of $E_{m}$ and $\omega$ were tested. As $E_{m}$ and $\omega$ increase, the elastic recovery (amount of strain recovered during unloading) decreases. The same calibration procedure was followed for MCC. Figure 6 shows comparison between experimental and simulation results for various values of the plastic, elastic, and bonding parameters. The difference between the simulation and experimental results are less for the unloading phase than that of the loading phase. Table 1 shows the best fitting $E_{m}$ and $\omega$ values for lactose and MCC.

One additional reason, among several possible reasons, for not getting perfectly matching curves may be the shape of particles. Only spherical particles are considered in the simulations, while the actual powders are composed of irregular particle shapes (Yohannes et al., 2015). Johansson and Alderborn (2001) have shown that the irregular shape of particles cause complex compression behavior, attrition of the particles, and increased bed void, which ultimately change the compaction profile. However, it is assumed 
that the differences between the simulations and experiments do not have a significant influence on the microstructure of the materials, especially for spherical particles that are used in this research.

Additional compaction simulations were done in order to analyze the effect of the ballistic deposition and particle size distribution on the compaction profile. Fig. 5.b shows comparison of compaction profiles for three samples. Sample 1 is the same as the results shown in Fig. 5.a. Sample 2 has the same particle size distribution and material properties as that of Sample 1, but different initial particles arrangement. Sample 3 has the same material properties as Sample 1, but different particle size distribution (particle size distribution shown in Fig. 2.b). The compaction profiles for all the samples are almost identical. These results are consistent with the experimental results (Fig. 1): the effects of the particle size distribution and the initial deposition of the powders on the compaction profile are negligible.

To validate the simulations, the tensile strength $\left(\sigma_{t}\right)$ of the tablets was measured and compared to that of the experiments. Using a diametrical tensile strength test (also known as indirect tensile strength test), in which a compression force is applied along the radial direction, the breaking force $\left(F_{\max }\right)$ for the tablets was measured. The tensile strength $\sigma_{t}$ can be computed from $F_{\max }$ as

$$
\sigma_{t}=\frac{2 F_{\max }}{\pi D t},
$$

where $D$ and $t$ are the diameter and thickness of the tablet, respectively. Fig. 7 shows the simulation set up and the measured diametrical force and strain for lactose tablets with out-of-die relative density (relative density after ejection) of about 0.94 . In the experiments, $F_{\max }$ for the tablets was measured 
using a hardness tester (Dr. Schleuniger Hardness Tablet Tester, Pharmatron) (Yohannes et al., 2015). Fig. 7 shows comparison of experimental measurements and simulation results of $\sigma_{t}$ at different levels of compaction. The simulation results are in good agreement with the experimental results. Since there was no material property calibration for the tensile strength simulation, these results indicate the calibrated material properties and the simulation procedure were appropriate. These simulations were run on Xeon(R) X5650 @ $2.67 \mathrm{GHz}$ processor with 12 cores and a simulation for one tablet (including deposition, compaction, ejection, and tensile strength test) takes about 72 hours wall time.

So far, a framework built upon clear physical theories based on the interparticle interactions was provided. It was demonstrated that the simulations can reproduce experimental results, such as compaction profiles and diametrical tensile strength, at various relative densities. These macroscopic predictions are dependent on the evolution of the microstructure which exhibits anisotropy patterns consistent with the experimental data. In the following section, the microstructure of Sample 1 is discussed in detail in terms contact area, magnitude and orientation of contact forces, and bonding between particles, along with some observations based on the other samples.

\section{Evolution of Microstructure}

As the relative density increases during the application of compaction pressure, the microstructure of the powder bed evolves. For example, coordination number has been shown to directly correlate with the compaction pressure (Olsson and Larsson, 2013; Sheng et al., 2004). Another measure 
of the microstructure during powder compaction is the inter-particle contact area. For tablet compaction applications, where the particles can bond with each other, the contact area between particles can be used as a partial measure of the strength of the compacts (Adolfsson et al., 1997) (Eq. 4). Fig. 8 shows the summation of all inter-particle contact areas between particles (total contact area) during loading and unloading of the compaction pressure for both lactose and MCC. For both materials, the total contact area increases with relative density during loading phase, and decreases slightly as the compaction pressure is gradually removed. The total contact areas for lactose and MCC are similar during the initial stages of compaction, up to relative density of about 0.6. A relative density of 0.60 is slightly less than random close packing (RCP) of cohesionless, spherical particles, which is about 0.64 (Scott and Kilgour, 1969). For relative densities below the RCP value, the compaction is dominated by particle position rearrangements rather than particle deformation. This could be one of the reasons for the similarity of contact areas of the lactose and MCC particles. Beyond relative density of 0.6 , the contact area for MCC is larger than that of lactose. The contact area for MCC is larger because MCC has lower plastic, elastic, and bonding parameters than that of lactose (Table 1). Larger contact area does not necessarily indicate higher tensile strength, particularly when comparing two different materials.

To understand the bond strength between particles, it is important to track the history/evolution of the magnitude of the contact forces between the particles during compaction and ejection. The evolution of the magnitude of all contact forces is best represented by plotting the probability distribution 
function (pdf), $f(P)$, of the contact forces. Fig. 9 and 10 show the pdf of the contact forces during the compaction, unloading, and ejection stages of the tablet compaction for lactose and MCC, respectively. Since the particles can form bonds between each other, some of the contact forces are in tension. (The tensile forces are represented by a negative sign in the Figures). The shape of the pdf and the magnitude of contact forces for lactose and MCC are similar. It is also noted that the distribution of forces for the samples with the same particle distribution (Sample 1 and Sample 2 in Fig. 5.b) are similar. However, for Sample 3, which contains more large particles, larger contact forces were observed. As the powder bed is compacted to higher relative densities, the pdf gets more positively skewed. The skewness indicates that the heterogeneity in the microstructure increases as the compaction force is increased. Since the bonding between particles is directly related to the contact forces between the particles, the heterogeneity will continue to exist even after the tablet is ejected from the die (Ragnar et al., 1995).

About $78 \%$ of all the contact forces are in compression at relative density of 1.0 and tensile contacts exist even during the application of the compaction pressure. The magnitude of the compression forces decreases as the compaction pressure is unloaded, while the limit for the tensile forces (about $-2 \mathrm{~N}$ ) remains the same during loading, unloading, and ejection. The distribution at the end of the unloading stage resembles a Gaussian distribution with a mean value of about zero. At the end of the unloading stage, the percentage of contact forces in compression reduces to about $55 \%$, for both lactose and MCC. The pdf during and after ejection is very similar to the pdf at the end of unloading, only getting slightly narrower, indicating a very 
small change in the contact forces during ejection of the tablet.

Though the contact force distribution after ejection is dominated by near zero forces, it does not mean that there is no contact or bonding between the particles. Actually, the inter-particle bond generated by the compaction pressure can support a larger tensile force. The bond force $\mathcal{B}$ for each interparticle contact, which is the tensile force required to break the bond between the particles, depends on the material properties and the deformation of the particles. $\mathcal{B}$ can be computed by taking the derivative of Eq. 4 with respect to the contact radius $(a)$. Fig. 11 shows the bond force generated due to contact force between two $100 \mu \mathrm{m}$ of MCC and two $100 \mu \mathrm{m}$ lactose particles. For both materials, $\mathcal{B}$ increases rapidly at lower compressive contact forces and it approaches a limiting value as the contact force is increased further. As shown in Fig. 9 and 10, the limit to the tensile contact forces is about $-2 \mathrm{~N}$, which is very close to the computed $\mathcal{B}$ for a pair of $100 \mu \mathrm{m}$ particles. It can be concluded that at tensile forces of beyond $-2 \mathrm{~N}$, almost all bonds between a pair of particles break.

Moreover, Fig. 12 shows the distribution of $\mathcal{B}(f(\mathcal{B}))$ for relative densities of between 0.65 to 0.95 . At these levels of compaction the value of $\mathcal{B}$ ranges between 0 and $-2.5 \mathrm{~N}$. As the compaction level (relative density) increases, $f(\mathcal{B})$ shifts to the left indicating an increase in the mean value of $\mathcal{B}$. Increase in $\mathcal{B}$ directly contributes to higher tablet tensile strength. In addition, the width of the distribution of $\mathcal{B}$ increases as the relative density increases, since at higher relative density some larger compression contact forces are created. The increase in the width of $f(\mathcal{B})$ also indicates increase in heterogeneity of the microstructure. It is also important to notice that the change in $f(\mathcal{B})$ 
diminishes as the relative density increases, especially at relative densities above 0.85 . The converging $f(\mathcal{B})$ corresponds to a plateau region of the tensile strength at high compaction pressures (Fichtner et al., 2008; Adolfsson et al., 1997; Ragnar et al., 1995)

For each contact between particles, the bond persists only if the actual tensile force is smaller than the maximum tensile force that can be supported. Therefore, during tablet compaction and ejection, bonds between particles may form, break, and/or reform. Discrete particle simulations provide complete information of inter-particle contacts, and the evolution of bond formation and breakage can be tracked. For lactose, for example, about $12 \%$ of the contact forces at relative density of 1.0 vanish when the tablet is ejected. On the other hand, only about $0.5 \%$ new contacts are formed/reformed during unloading and ejection.

Besides the evolution and heterogeneity of contact forces and bond strength, application of external forces may induce anisotropy in granular systems (Majmudar and Haines-Nutt, 2005; Mehrabadi et al., 1982; Ragnar et al., 1995; Mattsson and Nyström, 2001). The amount of anisotropy in granular systems is usually quantified by the fabric tensor. The fabric tensor is a second order tensor computed from the unit vector along the line connecting the centers of two contacting particles (Dubujet and Dedecker, 1998). Hence, the fabric tensor can be represented by plotting the orientation of the inter-particle contact forces. For convenience, the orientation of the contact forces is presented in two viewpoints in polar coordinate. First, the projection of the orientation of contact forces on XY-Z plane is shown (Fig. 13 and 14). (The compaction pressure is applied along the Z-axis). Second, the projection of 
the orientation on X-Y plane is shown (Fig. 15 and 16). The orientation of the tensile and compression contact forces are shown separately. The radial direction indicates the magnitude of the projected force in units of Newton and the angular direction represents the angle the contact force makes with $\mathrm{XY}$-plane or X-axis.

Fig. 13 (a) and (b) show the orientation of the compressive contact forces and the tensile contact forces, respectively, on XY-Z plane for lactose at relative density of 1.0 (porosity of 0.0 ). The large compressive contact forces (with magnitudes reaching up to $10 \mathrm{~N}$ ) are mostly oriented along the direction of compaction pressure (along Z-axis), but only the small compressive forces (maximum magnitude of about $2 \mathrm{~N}$ ) are oriented along the XY-plane. On the other hand, the tensile forces are mostly oriented perpendicular to the direction of compaction. Almost all the tensile contact forces lie within \pm $30^{\circ}$ from the XY-plane. This is also true for the other samples, Sample 2 and Sample 3, but slightly larger contact forces develop during compaction in Sample 3. This anisotropy in the contact forces could be one of the reasons for the difference between the axial and diametrical tensile strengths observed in prior experiments (Ragnar et al., 1995; Mattsson and Nyström, 2001).

When the compaction pressure is removed, the orientation of the contact forces also changes. Fig. 14 shows the orientation of contact forces on the $\mathrm{XY}-\mathrm{Z}$ plane after ejection of a tablet. In general, the orientation of the compressive contact forces (Fig. 14(a)) and the tensile contact forces (Fig. 14(b)) after ejection of the tablet is more isotropic than during the maximum loading. The magnitude of compressive forces decrease after ejection, where as the increase in the tensile forces after ejection is negligible in all directions. 
The anisotropy of contact forces generated during the loading phase vanishes after the ejection of the tablet from the die. However, it is important to note that large bonding forces (maximum tensile force) are created due to the large compressive contact forces. The isotropic contact forces after ejection by no means indicate an isotropic bonding force between particles. The orientation of contact forces, during compaction and after ejection, on the $\mathrm{X}-\mathrm{Y}$ plane is isotropic for both the compression and tensile contact forces (Fig. 15 and Fig. 16). The diametrical tensile strength of a tablet would be the same independent the direction of breaking force on the X-Y plane.

\section{Conclusion}

Discrete particle modeling is an ideal tool to investigate the microstructure evolution in powder compaction applications. In this study, a discrete particle model that accounts for plastic, elastic, and bonding properties of the particles, in addition to representing the exact particle size distribution of lactose and MCC powders, was used. During compaction the initial contact force between particle is compressive. However as the particle rearrange and deform some of the contact forces develop to a tensile forces, and eventually very few of these contacts break. As the compaction pressure is increased, the positive skewness of the probability distribution function of the contact forces increases, dominated by compressive contact forces. Whereas, the maximum value of the tensile contact forces remains within the limits of the bond force $\mathcal{B}$. During unloading of the compaction pressure, the distribution approaches a normal distribution with a mean value of zero. This evolution of contact forces is accompanied by evolution of the anisotropy in the pow- 
der bed. Particularly, during application of compaction pressure, the largest compressive contact forces are aligned along the direction of the compaction pressure, whereas the tensile contact forces are oriented perpendicular to the compaction pressure. After ejection, the contact forces becomes isotropic.

The simulations clearly show the evolution of the microstructure during tablet compaction. Anisotropy, and hence inhomogeneity, is generated in tablets due to the compaction process itself. The anisotropy will cause directional preference of breakage and cracks, and capping tendencies in tablets. Understanding the evolution and nature of the contact forces due to the compaction pressure is essential to mitigate these problems caused by the heterogeneity and anisotropy in tablets. One possible way is to design shape of the tooling that reduces the anisotropy in the particle-particle contact forces. The numerical models introduced in this manuscript can play a significant role towards selecting appropriate tooling for powder compaction.

\section{Acknowledgement}

We gratefully acknowledge the funding for this research provided by Bristol-Myers Squibb Company. We also acknowledge the support provided by the National Science Foundation Engineering Research Center for Structured Organic Particle Systems (C-SOPS).

\section{References}

Adolfsson, Å., Olsson, H., and Nyström, C., 1997. Effect of particle size and compaction load on interparticulate bonding structure for some pharma- 
ceutical materials studied by compaction and strength characterisation in butanol. European Journal of Pharmaceutics and Biopharm. 44, 243-251.

Almaya, A. and Aburub, A., 2008. Effect of particle size on compaction of materials with different deformation mechanisms with and without lubricants. AAPS PharmSciTech. 9(2), 414-418.

Bratberg, I., Radjai, F., and Hansen, A., 2002. Dynamic rearrangements and packing regimes in randomly deposited two-dimensional granular beds. Phys. Rev. E 66, 031303.

Castellanos, A., 2005. The relationship between attractive interparticle forces and bulk behaviour in dry and uncharged fine powders. Advances in Physics 54(4), 263-376.

Cundall, P. A. and Strack, O. D. L., 1979. A discrete numerical model for granular assemblies. Geotech. 29, 47-65.

Dubujet, P. and Dedecker, F., 1998. Micro-mechanical analysis and modelling of granular materials loaded at constant volume. Granular Matter 1(3), $129-136$.

Eichie, F. E. and Kudehinbu, A. O., 2009. Effect of particle size of granules on some mechanical properties of paracetamol tablets. African Journal of Biotechnology 8(21), 5913-5916.

Fichtner, F., ke Rasmuson, A., and Alderborn, G., 2005. Particle size distribution and evolution in tablet structure during and after compaction. International Journal of Pharmaceutics 292, 211 - 225. 
Fichtner, F., Mahlin, D., Welch, K., Gaisford, S., and Alderborn, G., 2008. Effect of Surface Energy on Powder Compactibility. Pharmaceutical Research 25(12), $2750-2759$.

Gonzalez, M. and Cuitiño, A. M., 2012. A nonlocal contact formulation for confined granular systems. Journal of the Mechanics and Physics of Solids $60(2), 333-350$.

Gonzalez, M. and Cuitiño, A. M., 2015. Microstructure evolution of compressible granular systems under large deformations. Journal of the Mechanics and Physics of Solids under review.

Güner, F., mer Necati Cora, and Sofuolu, H., 2015. Numerical modeling of cold powder compaction using multi particle and continuum media approaches. Powder Technology 271(0), $238-247$.

Heckel, R. W., 1961. Density-pressure relationships in powder compaction. Transactions of the metallurgical Society of AIME 221, $671-675$.

Herting, M. G. and Kleinebudde, P., 2007. Roll compaction/dry granulation: Effect of raw material particle size on granule and tablet properties. International Journal of Pharmaceutics, 338, 110-118.

Johansson, B. and Alderborn, G., 2001. The effect of shape and porosity on the compression behaviour and tablet forming ability of granular materials formed from microcrystalline cellulose. European Journal of Pharmaceutics and Biopharmaceutics 52(3), $347-357$.

Jullient, R., Pavlovitch, A., and Meakin, P., 1992. Random packings of 
spheres built with sequential models. J. Phys. A Math. Gen. 25, 41034113.

Khomane, K. S. and Bansal, A. K. (2013). Effect of particle size on in-die and out-of-die compaction behavior of ranitidine hydrochloride polymorphs. AAPS PharmSciTech. 14(3), 1169-1177.

Klinzing, G. R., Zavaliangos, A., Cunningham, J., Mascaro, T., and Winstead, D., 2010. Temperature and density evolution during compaction of a capsule shaped tablet. Computers and Chemical Engineering 34(7), 1082 $-1091$.

Koynov, A., Akseli, I., and Cuitiño, A., 2011. Modeling and simulation of compact strength due to particle bonding using hybrid discrete-continuum approach. International Journal of Pharmaceutics 418, 273 - 285.

Majmudar, N. A. and Haines-Nutt, R. F., 2005. Contact force measurements and stress-induced anisotropy in granular materials. Nature 435, 10791082.

Martin, C. and Bouvard, D. (2004). Isostatic compaction of bimodal powder mixtures and composites. International Journal of Mechanical Sciences 46(6), $907-927$.

Mattsson, S. and Nyström, C., 2001. Evaluation of Critical Binder Properties Affecting the Compactibility of Binary Mixtures. Drug Development and Industrial Pharmacy 27(3), 181- 194. 
Mckenna, A. and Mccafferty, D. F., 1982. Effect of particle size on the compaction mechanism and tensile strength of tablets. Journal of Pharmacy and Pharmacology 34(6), 347-351.

Mehrabadi, M. M., Nemat-Nasser, S., and Oda, M., 1982. On statistical description of stress and fabric in granular materials. International Journal for Numerical and Analytical Methods in Geomechanics 6(1), 95-108.

Mesarovic, S. D. and Johnson, K., 2000. Adhesive contact of elasticplastic spheres. Journal of the Mechanics and Physics of Solids, 48(10), 2009-2033.

Mueller, G. E., 1997. Numerical simulation of packed beds with monosized spheres in cylindrical containers. Powder Technology 92(2), 179 - 183.

Olsson, E. and Larsson, P.-L., 2013. A numerical analysis of cold powder compaction based on micromechanical experiments. Powder Technology $243(0), 71-78$.

Patel, S., Kaushal, A., and Bansal, A., 2007. Effect of particle size and compression force on compaction behavior and derived mathematical parameters of compressibility. Pharmaceutical Research 24(1), 111-124.

Potyondy, D. O. and Cundall, P. A., 2004. A bonded-particle model for rock. Int. J. Rock Mech. and Min. Sci. 41(8), 1329-1364.

Ragnar, E. K., Wormald, P., Östelius, J., Iversen, T., and Nyström, C., 1995. Crystallinity index of microcrystalline cellulose particles compressed into tablets. International Journal of Pharmaceutics 125, 257- 264. 
Scott, G. D. and Kilgour, D. M., 1969. The density of random close packing of spheres. Journal of Physics D: Applied Physics 2(6), 863.

Sheng, Y., Lawrence, C., Briscoe, B., and Thornton, C., 2004. Numerical studies of uniaxial powder compaction process by $3 \mathrm{~d}$ dem. Engineering Computations, 21(2/3/4), 304-317.

Skrinjar, O. and Larsson, P.-L., 2004. Cold compaction of composite powders with size ratio. Acta Materialia 52(7), 1871 - 1884.

Storåkers, B., Biwa, S., and Larsson, P.-L., 1997. Similarity analysis of inelastic contact. International Journal of Solids and Structures 34(24), $3061-3083$.

Storåkers, B., Fleck, N. A., and McMeeking, R. M., 1999. The viscoplastic compaction of composite powders. Journal of the Mechanics and Physics of Solids 47(4), $785-815$.

Sun, C. C., 2008. On the mechanism of reduced tabletability of granules prepared by roller compaction. International Journal of Pharmaceutics $347(1-2), 171-172$.

Sun, C. C. and Himmelspach, M. W., 2006. Reduced tabletability of roller compacted granules as a result of granule size enlargement. Journal of Pharmaceutical Sciences 95(1), 200-206.

Yohannes, B., Gonzalez, M., Abebe, A., Sprockel, O., Nikfar, F., Kiang, S., and Cuitino, A., 2015. The role of fine particles on compaction and tensile strength of pharmaceutical powders. Powder Technology 274(0), 372 378. 
Zavaliangos, A., 2002. A multiparticle simulation of powder compaction using finite element discretization of individual particles. In Symposium W Modeling and Numerical Simulation of Materials Behavior and Evolution volume 731 .

Zhang, J., 2009. A study of compaction of composite particles by multiparticle finite element method. Composites Science and Technology 69(13), $2048-2053$.

Zhang, J. and Zavaliangos, A., 2011. Discrete finite-element simulation of thermoelectric phenomena in spark plasma sintering. Journal of Electronic Materials 40(5), 873-878.

Zheng, S. and Cuitiño, A. M., 2002. Consolidation behavior of inhomogeneous granular beds of ductile particles using a mixed discrete-continuum approach. KONA Powder and Particle Journal 20, 168-177.

Zou, R. P. and Yu, A. B., 1996. Wall effect on the packing of cylindrical particles. Chemical Engineering Science 51(7), 1177-1180. 
TABLE

Table 1: Material physical properties

\begin{tabular}{|l|c|c|c|c|}
\hline Powder & $\mathrm{k}(\mathrm{MPa})$ & $\mathrm{m}$ & $\mathrm{E}(\mathrm{GPa})$ & $\omega\left(\mathrm{J} / \mathrm{m}^{2}\right)$ \\
\hline Lactose & 3750.0 & 1.0 & 10.0 & 1100.0 \\
\hline MCC & 150.0 & 4.0 & 4.0 & 500.0 \\
\hline
\end{tabular}




\section{FIGURES}

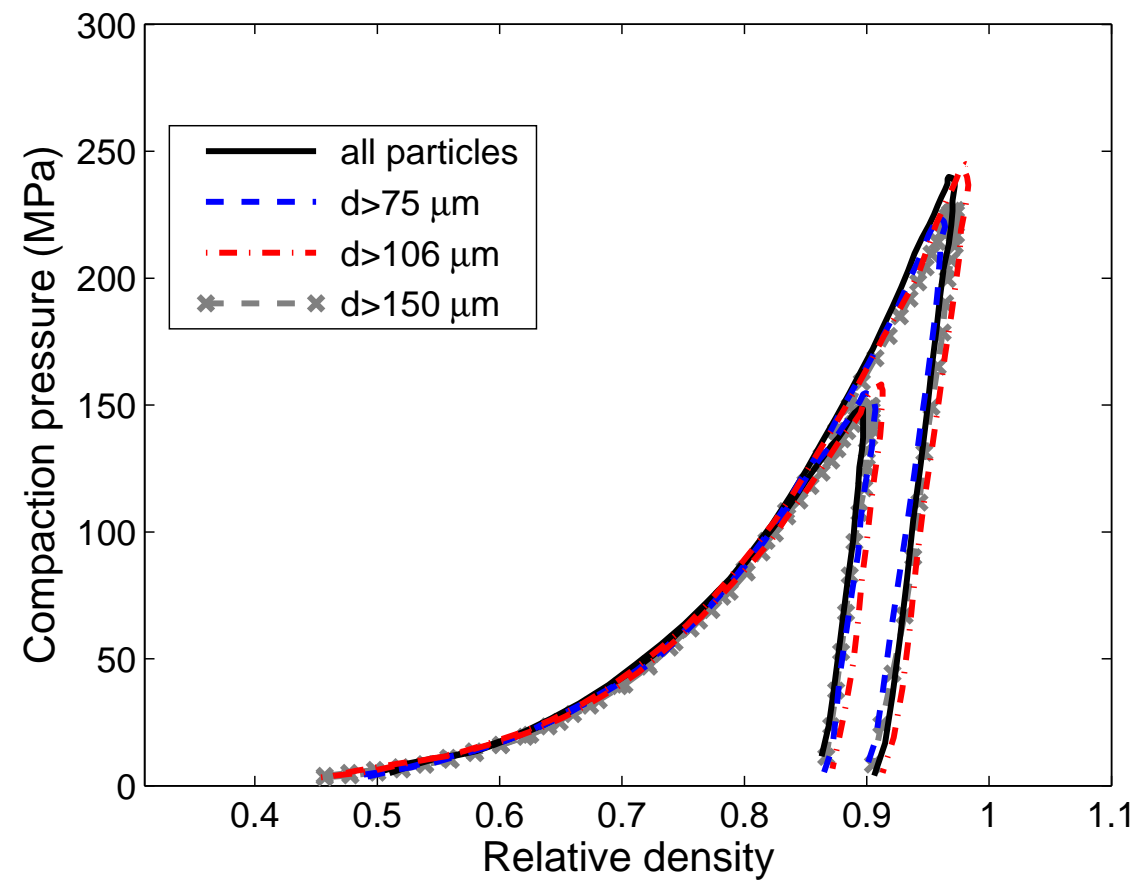

Figure 1: Compaction pressure vs. Relative density for lactose samples with different particle size distribution. 

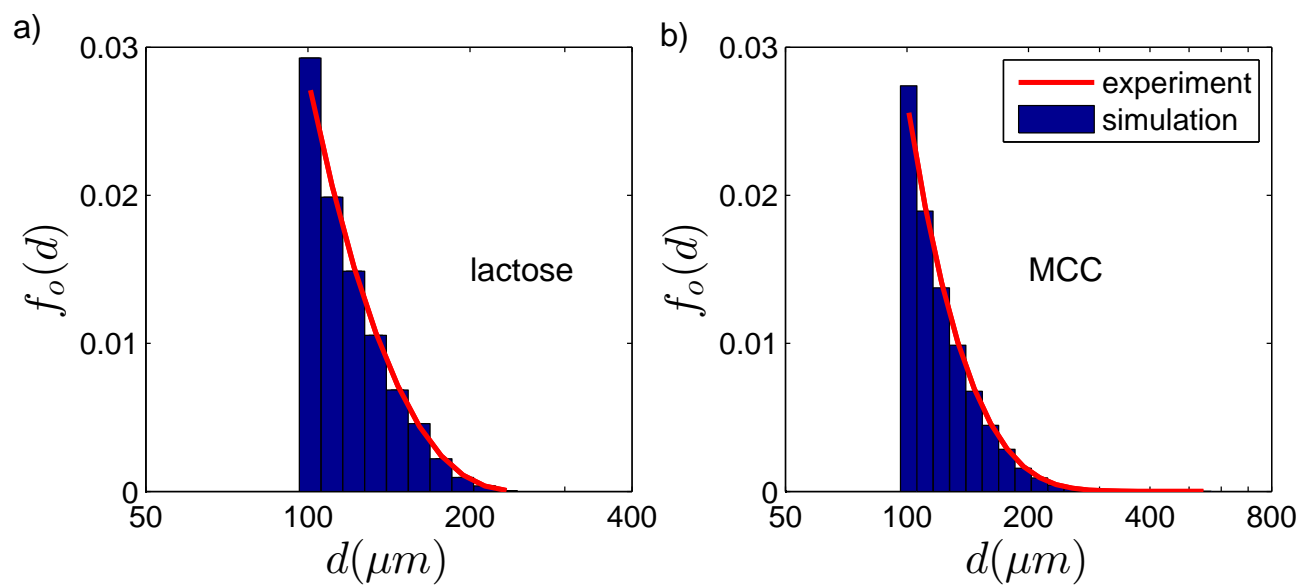

Figure 2: Particle size distribution based on the number of particles for (a) lactose and (b) MCC. The horizontal axis is in logarithmic scale. 

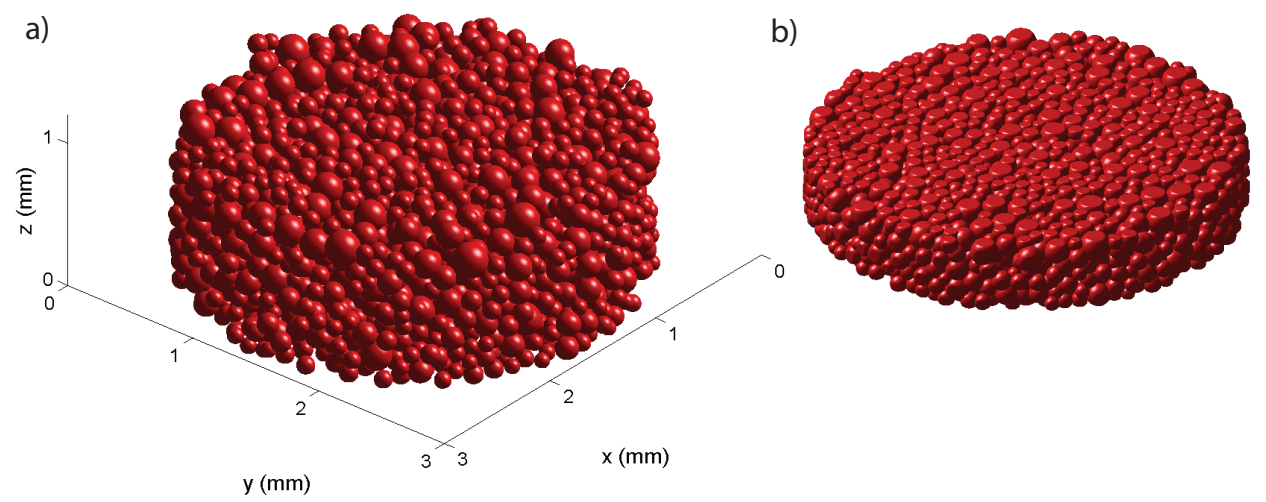

Figure 3: a)Powder bed of lactose particles after ballistic deposition. b)compacted lactose powder (relative density $\approx 0.9$ ). 


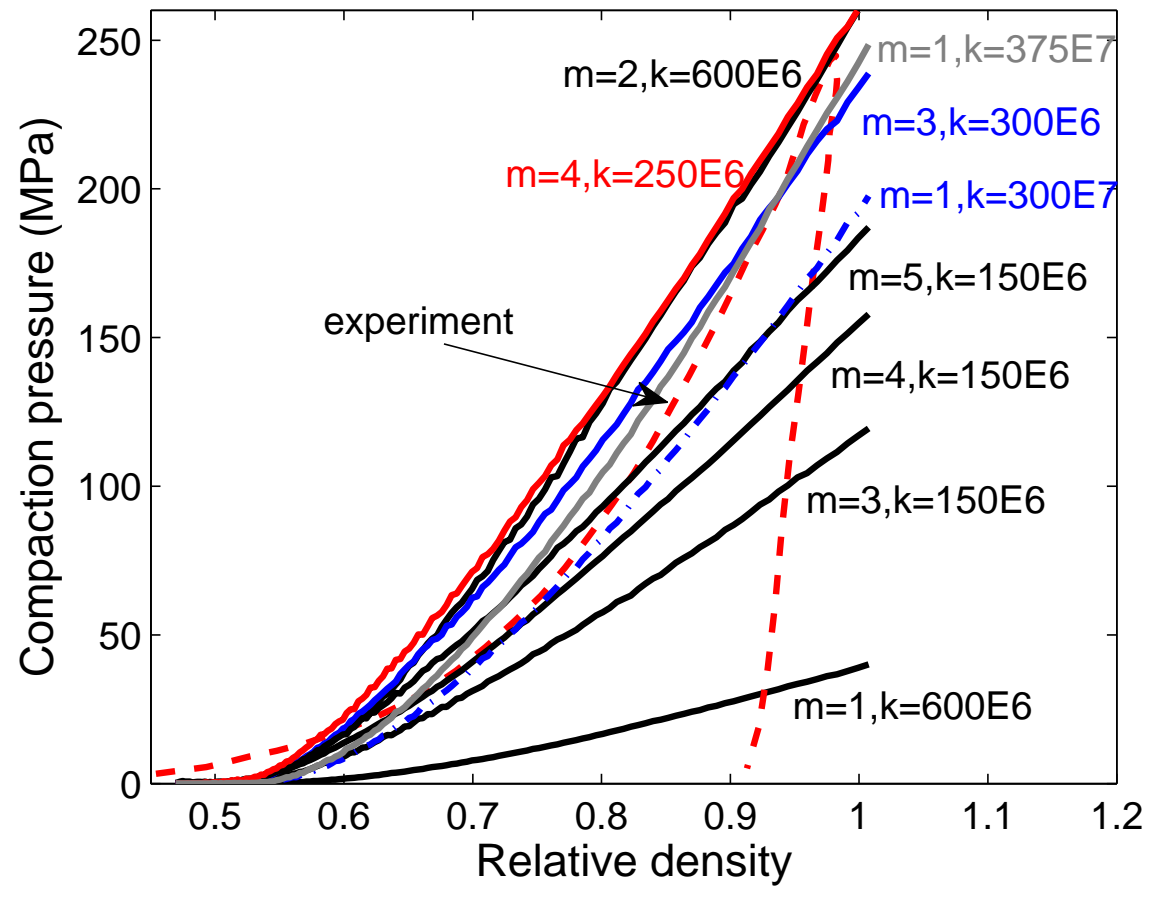

Figure 4: Calibration of plastic parameter for lactose. (The units of $k$ is $P a$ ). The best fitting values are $k=3.75 \mathrm{GPa}$ and $m=1.0$. 
a)

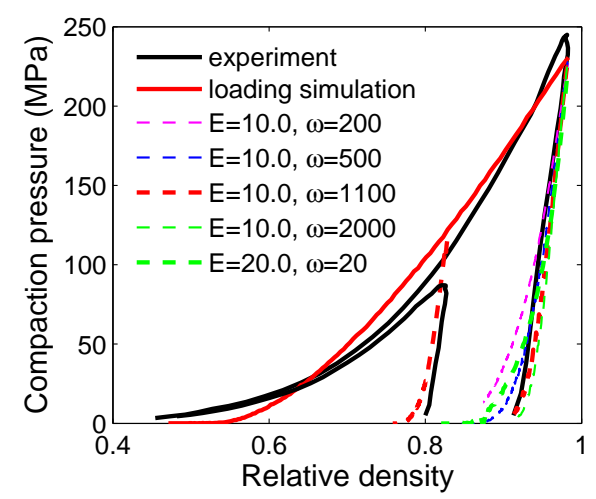

b)

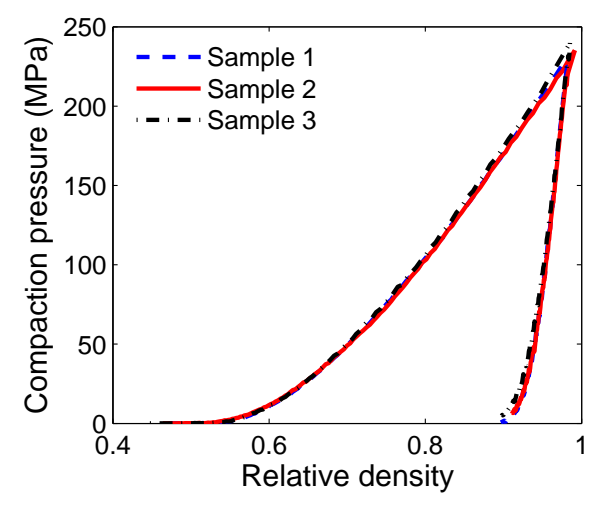

Figure 5: a) Calibration of Elastic $(E)$ and Bonding $(\omega)$ parameters for lactose. The best fitting simulation is for $E=10 \mathrm{GPa}$ and $\omega=1100 \mathrm{~J} / \mathrm{m}^{2}$. b) Comparison of compaction profile results from simulations for three different lactose samples. 


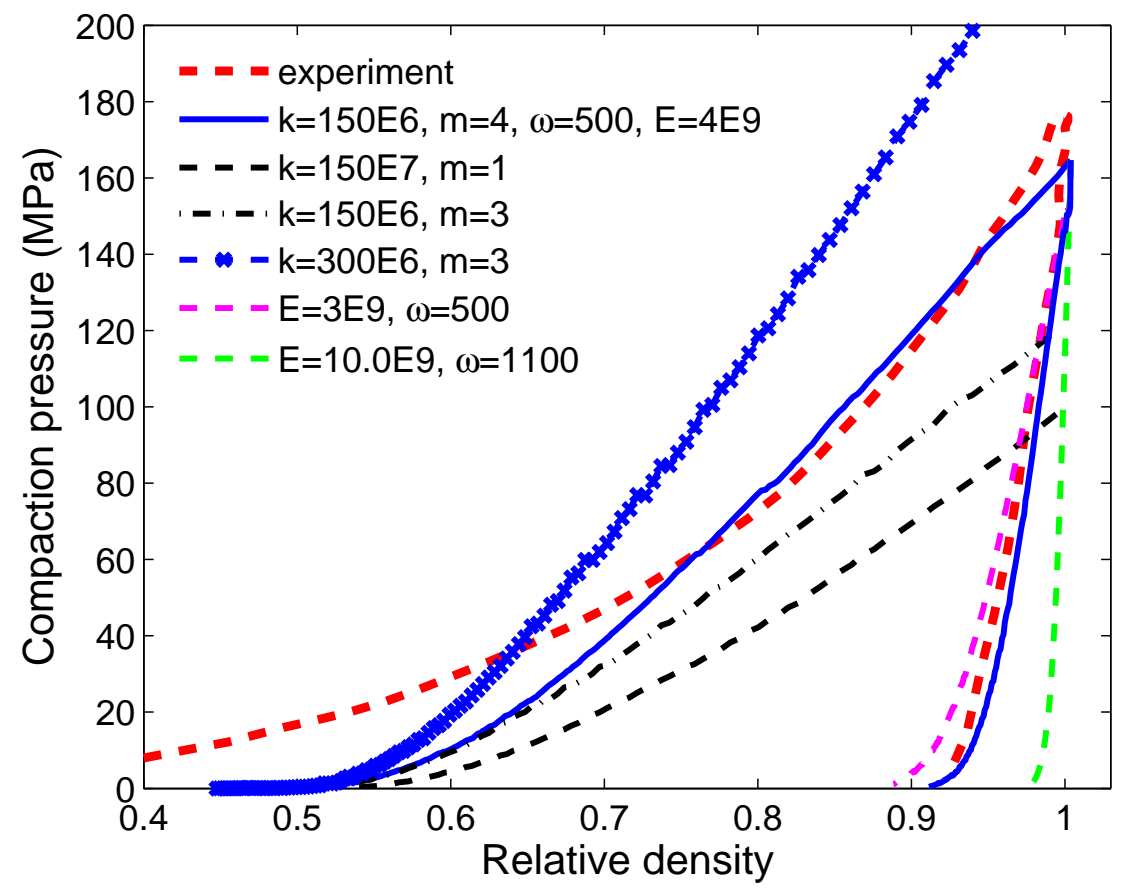

Figure 6: Comparison of experimental and simulation results for MCC. $k$ and $E$, are given in $P a$ and $\omega$ is in $\mathrm{J} / \mathrm{m}^{2}$. The best fitting values are $k=150 \mathrm{MPa}, m=4, E=4.0 \mathrm{GPa}$, and $\omega=500 \mathrm{~J} / \mathrm{m}^{2}$. 

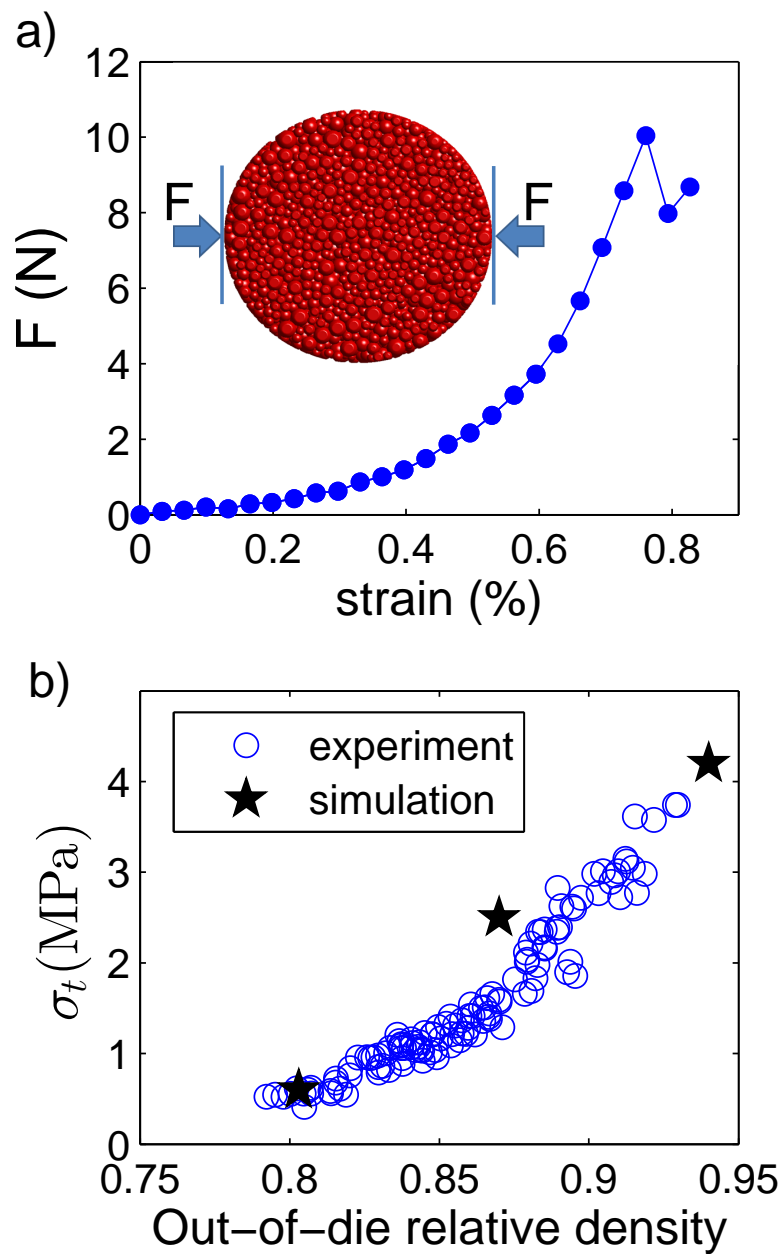

Figure 7: a) Force vs. strain measured during a diametrical tensile strength test for lactose. The test set up is shown in the inset. b)Tensile strength $\left(\sigma_{t}\right)$ vs. out-of-die relative density (relative density of a tablet after ejection) plot from experiments and simulations for lactose. 


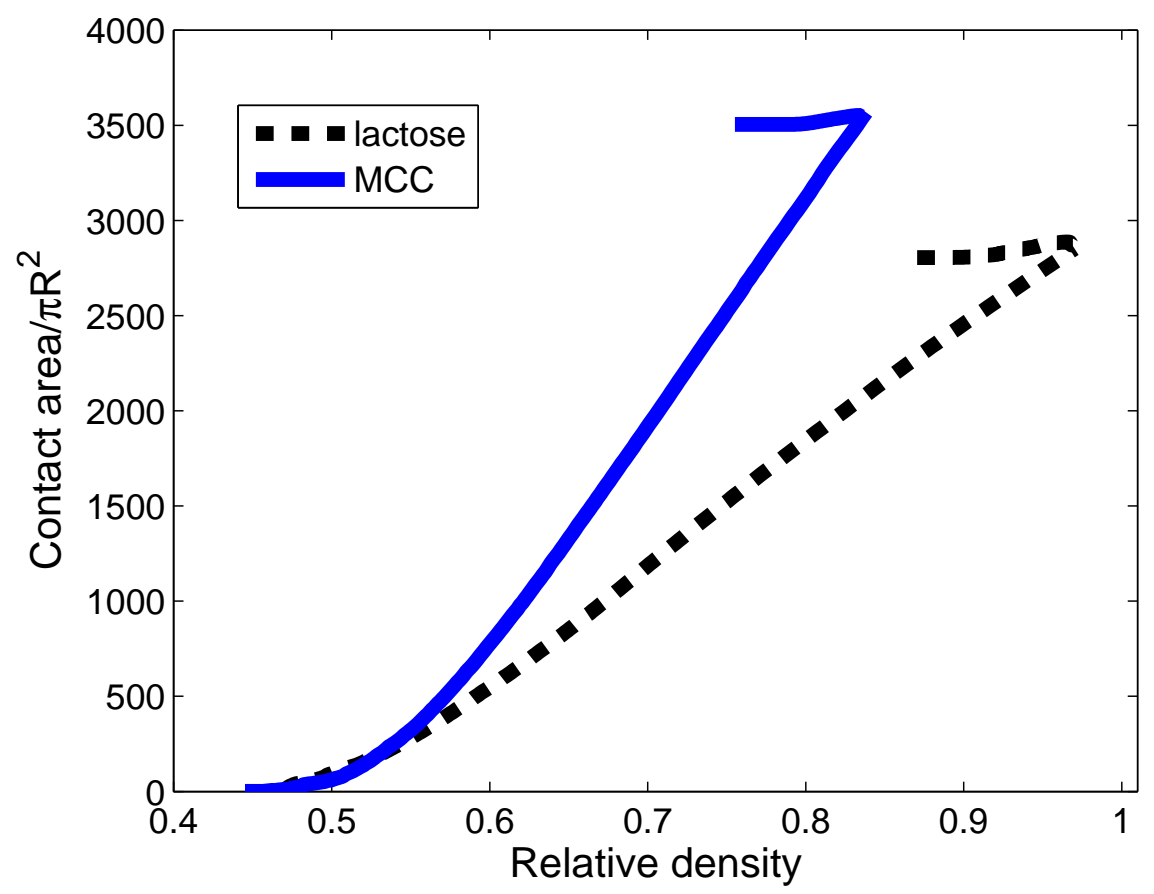

Figure 8: Sum of all contact areas vs. relative density, during compaction, unloading, and ejection. The contact areas is normalized by the projected area of the smallest particle in the sample. 


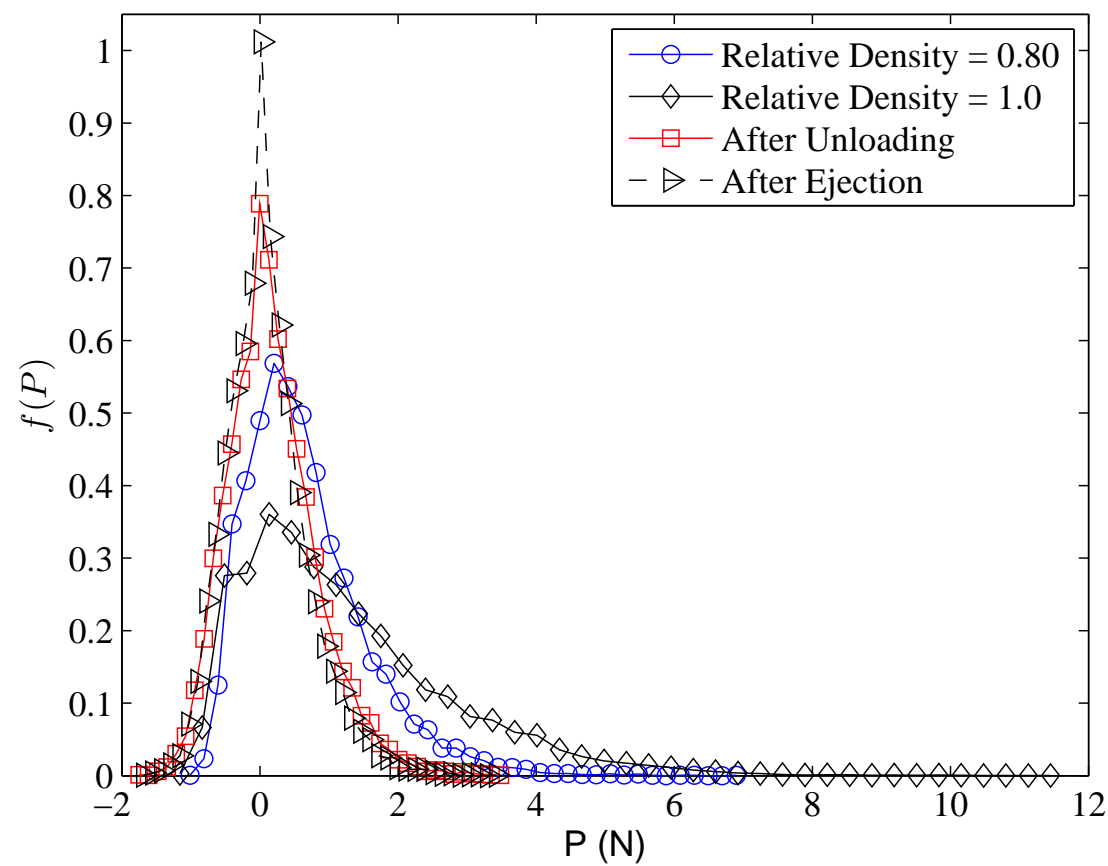

Figure 9: Probability distribution for contact forces during different stages of tablet compaction for lactose. 


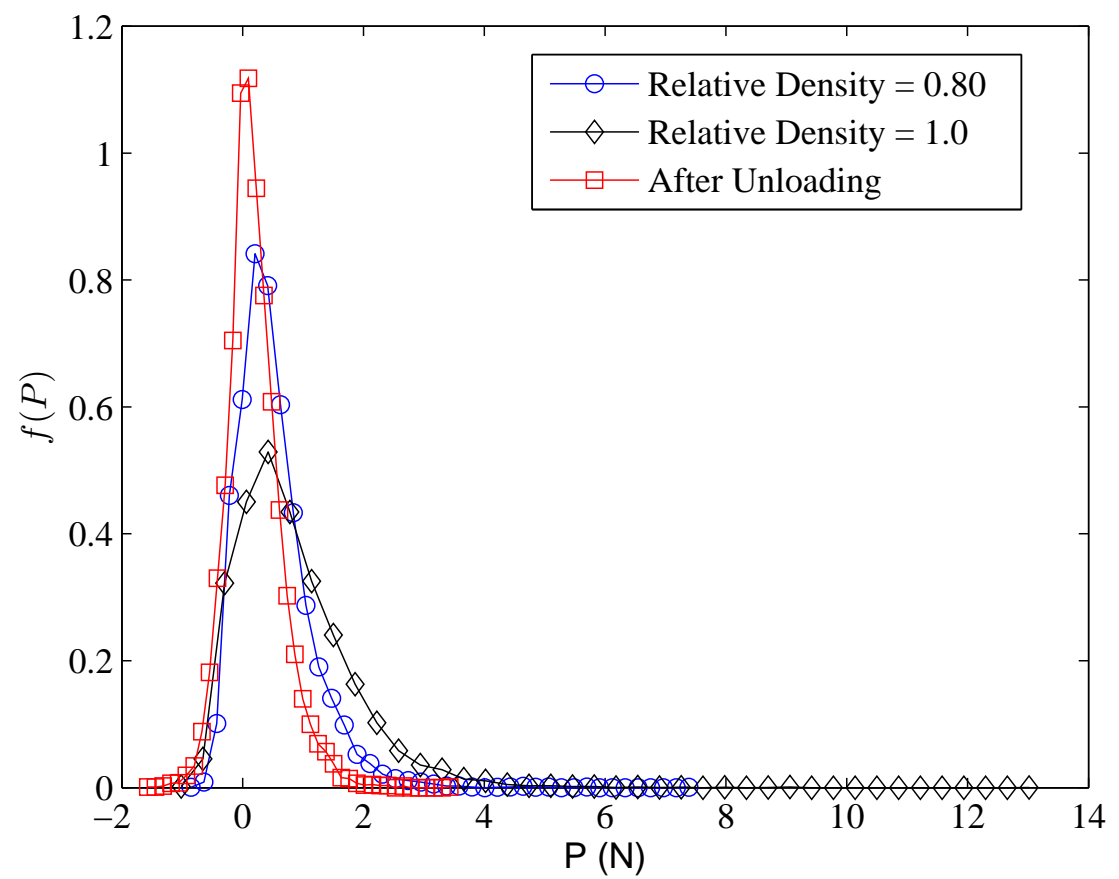

Figure 10: Probability distribution for contact forces during different stages of tablet compaction for MCC. 


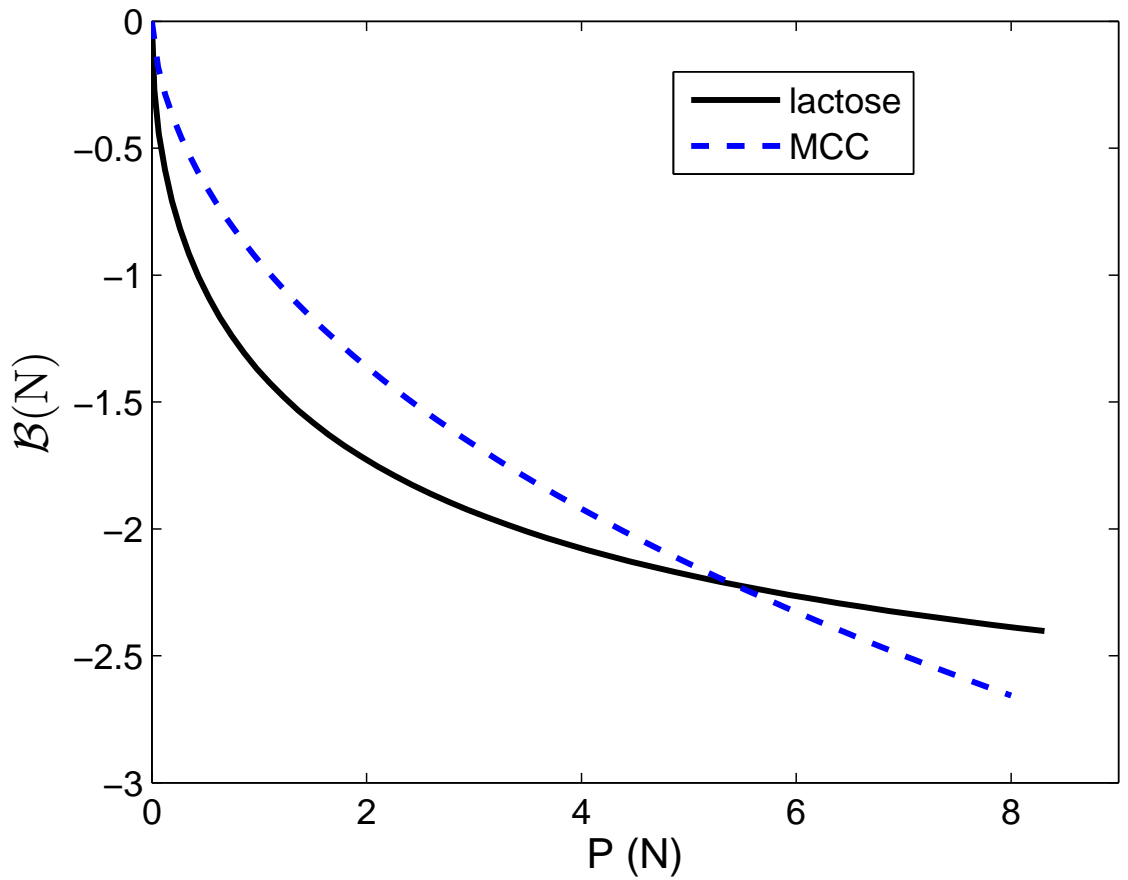

Figure 11: The maximum tensile strength generated due to a contact force between two $100 \mu \mathrm{m}$ particles. 


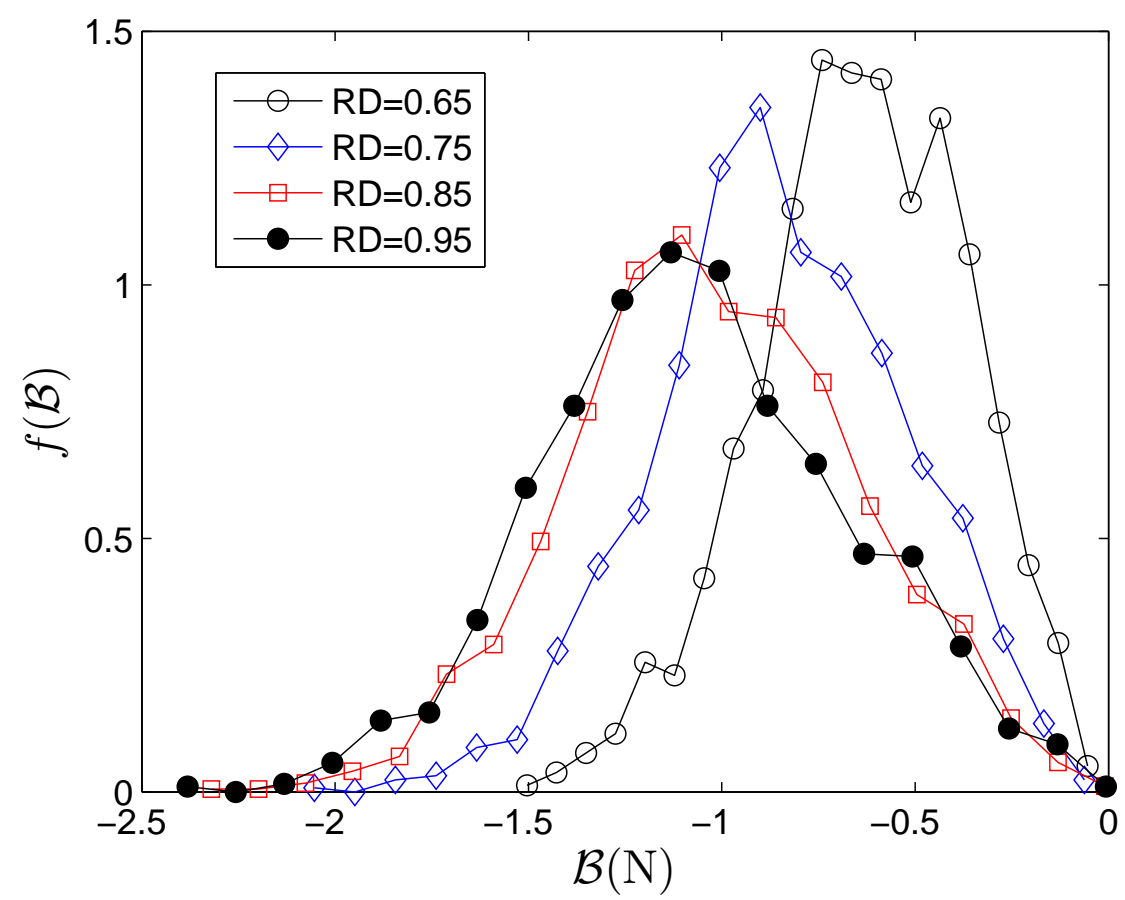

Figure 12: Probability distribution of maximum tensile force that can be supported by each particle-particle contact for lactose at different levels of compaction. 
a)

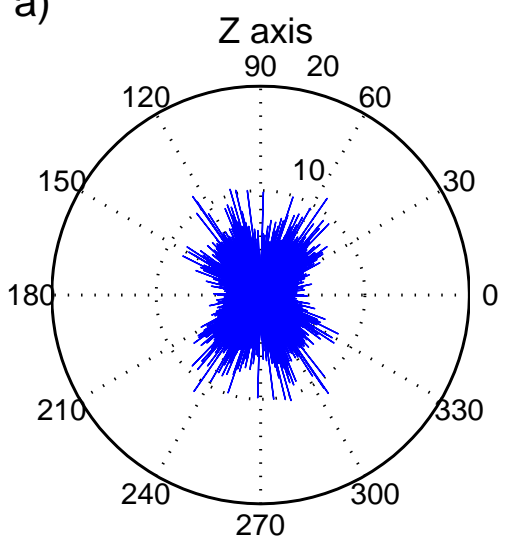

b) $\quad Z$ axis

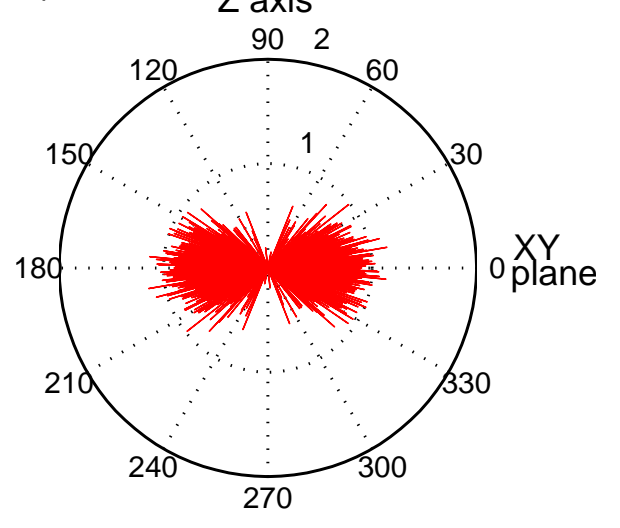

Figure 13: Orientation of contact forces on the XY-Z plane during compaction at relative density of 1.0 . (a) Orientation of compressive contact forces (b) Orientation of tensile contact forces. The compaction force is applied along the Z-axis. 

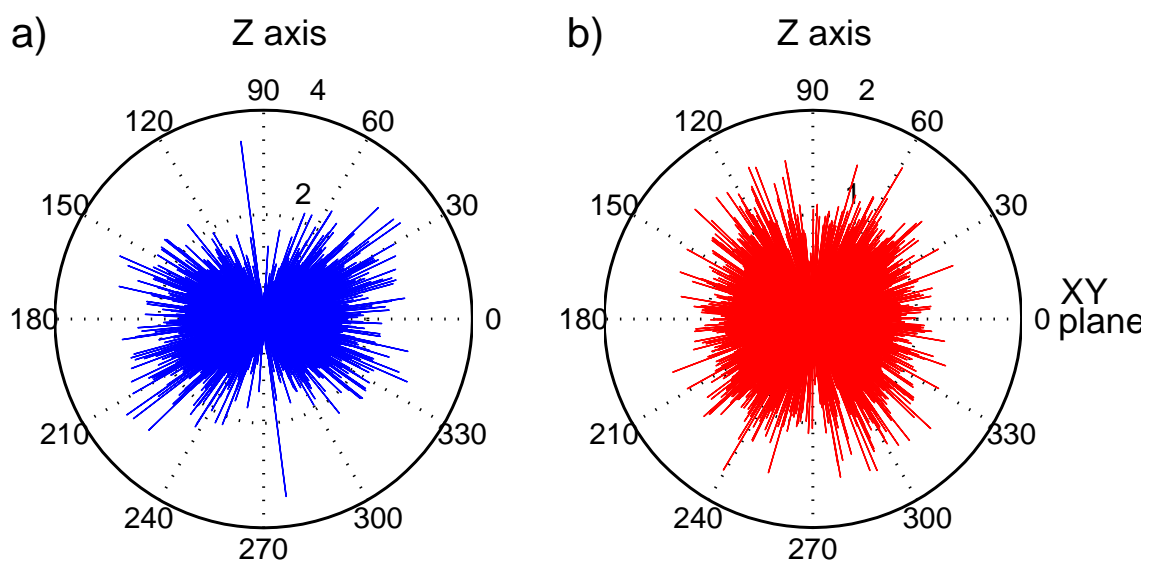

Figure 14: Orientation of contact forces on the XY-Z plane after ejection (a) Orientation of compressive contact forces (b) Orientation of tensile contact forces. The compaction force is applied along the Z-axis. 

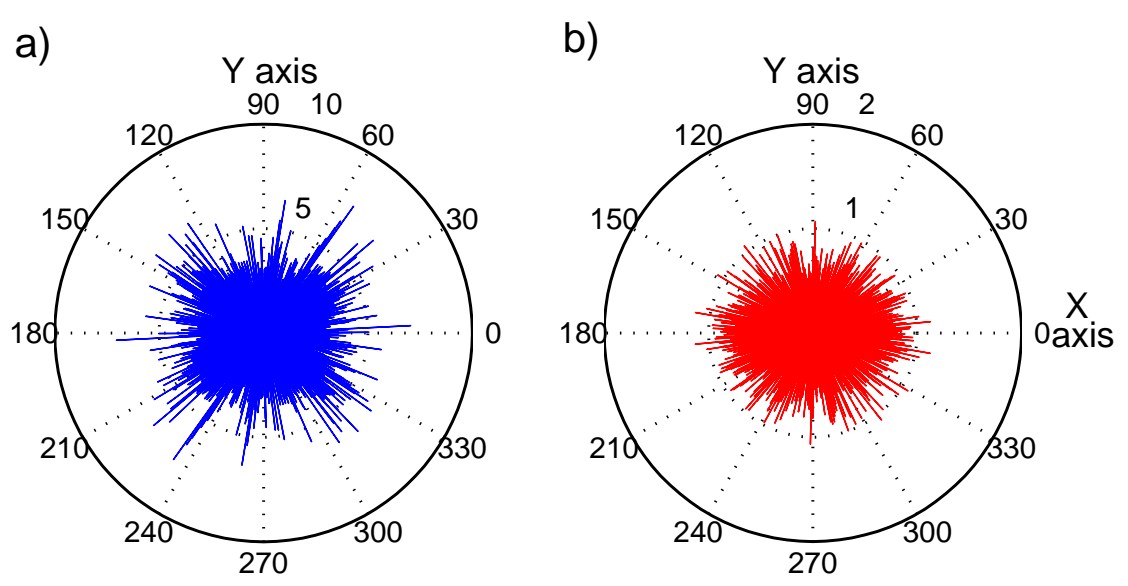

Figure 15: Orientation of contact forces on the $\mathrm{X}-\mathrm{Y}$ plane during compaction at relative density of 1.0 (a) Orientation of compressive contact forces (b) Orientation of tensile contact forces. The compaction force is applied along the Z-axis. 

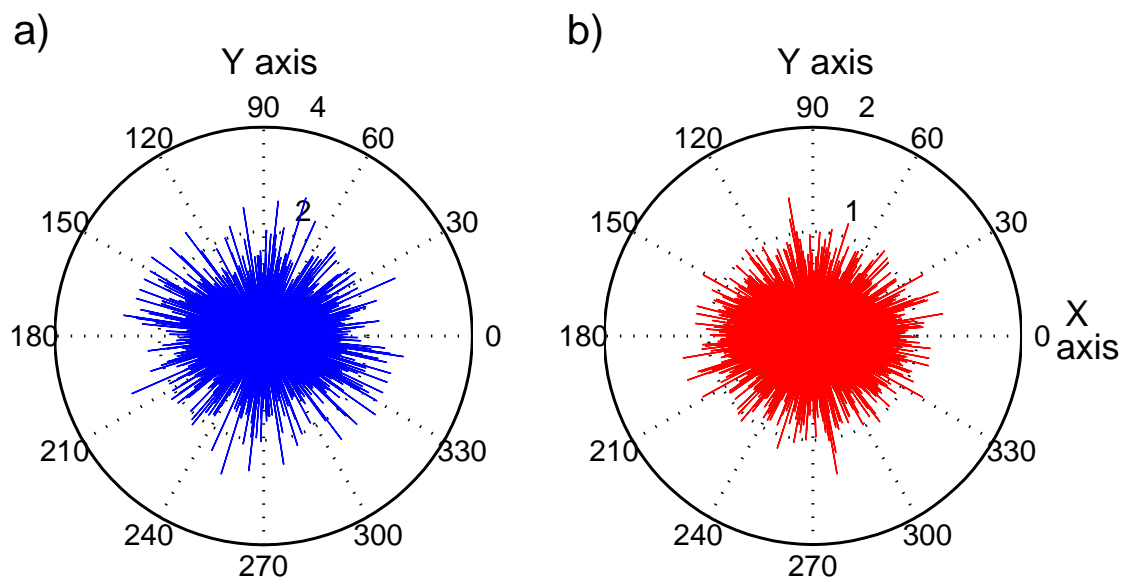

Figure 16: Orientation of contact forces on the X-Y plane after ejection (a) Orientation of compressive contact forces (b) Orientation of tensile contact forces. The compaction force is applied along the Z-axis. 


\section{Graphical Abstract}

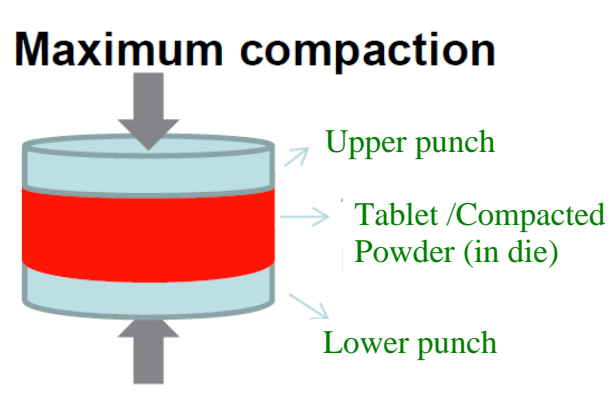

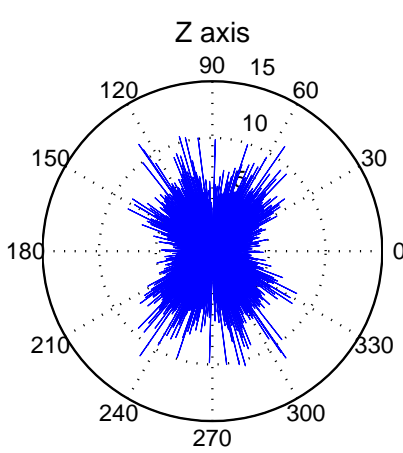

Compression forces

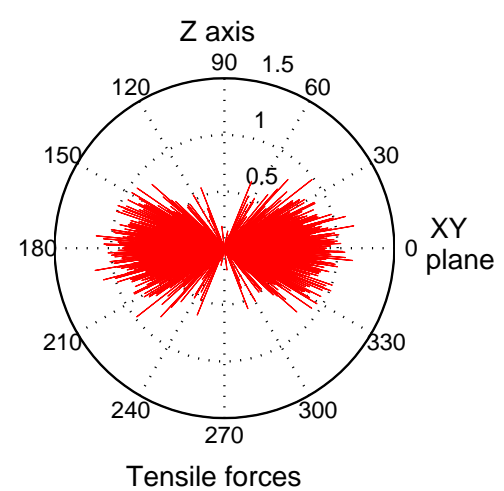

Tensile forces 